\title{
Reviewers for Volume 30
}

The following colleagues have generously given their time and energy to review papers for Law and Human Behavior in the last year. We are grateful for their contributions to this journal.

Alter, Adam L
Appelbaum, Paul
Arkes, Hal
Baldus, David
Berman, Garrett
Bersoff, Donald N
Blair, J. P.
Blumenthal, Jeremy A
Boccacinni, Marcus T
Boer, Douglas
Bond, Charles
Bond, Gary
Bornstein, Brian
Bottoms, Bette L
Bourgeios, Martin J
Brewer, Neil
Bruck, Maggie
Burke, Tara
Butler, Brooke
Candilis, Philip
Carlsmith, Kevin
Cecil, Joe
Chauhan, Preeti*
Clark, Steven
Connolly, Deborah Ann
Cook, James
Cooke, David
Cooper, Virginia Galloway

Cornell, Dewey

Craig-Henderson, Kellina M

Crossman, Angela M

Culhane, Scott E

Davis, Deborah

Deffenbacher, Kenneth A

Demakis, George

DeMatteo, David

Dennison, Susan M

Devenport, Jennifer

Devine, Dennis J

Diamond, Shari Seidman

Dillehay, Ronald C

Dobbin, Shirley A

Douglas, Kevin S

Douglass, Amy Bradfield

Dvoskin, Joel

Dysart, Jennifer

Edens, John

Eisenstadt, Donna

Elaad, Eitan

Elbogen, Eric Bradley

Exum, Lyn

Faigman, David L

Feigenson, Neal

Fisher, Ron

Flowe, Heather

Fondacaro, Mark R
ForsterLee, Lynne

Fulero, Soloman M

Gagliardi, Gregg J

Galietta, Michele

Gallo, Frank J

Gagnon, Nathalie*

Gardner, William

Geiselman, Edward

Gilstrap, Livia L

Goldstein, Naomi E

Gordon, Randall

Granhag, Par Anders

Greene, Edith

Groscup, Jennifer

Gudjonsson, Gisli

Gutek, Barbara

Hafemeister, Thomas L

Hagen, Margaret

Haney, Craig W

Harris, Grant

Hart, Stephen D

Haw, Ryann M

Henle, Christine

Heuer, Larry

Holtz, Brian C

Honts, Charles R

Hosch, Harmon

Hoskins, Stacy* 
Hunt, Jennifer S

Huss, Matthew T

Jackson, Rebecca L

Kassin, Saul

Kelloway, E. Kevin

Kerr, Norbert L

Krauss, Daniel A

Koenken, Guenter

Kostelnik, Jessica*

Kravitz, David

Lamb, Michael E

Lane, Sean

Lanyon, Richard

Larsson, Henrik

Lassiter, G Daniel

Lecci, Len

Leippe, Michael R

Lenhardt, Thomas*

Leo, Richard A

Levett, Lora

Levi, Avraham M

Lidz, Charles W

Lieberman, Joel

Lindsay, Rod C L

Linz, Daniel

Lord, Vivian

Lynch, Mona

MacCoun, Robert

MacLin, Kim

MacLin, Otto H

McQuiston-Surrett, Dawn

Malpass, Roy S

McAllister, Hunter

McAuliff, Bradley D

Meissner, Christian A

Memon, Amina

Mercado, Cynthia Calkins

Miller, Bryan

Mitchell, Tara

Monahan, John

Mossman, Douglas
Muehlenhard, Charlene

Myers, Bryan

Nicholls, Tonia L

Neuschatz, Jeffrey S

Nunez, Narina

O'Connor, Maureen

O'Neil, Kevin

O'Sullivan, Maureen

Olson, Elizabeth

Otto, Randy

Park, Jaihyun

Patry, Marc

Pezdek, Kathy

Pfeifer, Jeff E

Pickel, Kerri

Piquero, Nicole

Platania, Judith

Polage, Danielle

Pollina, Dean

Posey, Amy J

Porter, Stephen

Powell, Martine

Poythress, Norman

Pozzulo, Joanna

Quas, Jodi A

Rachlinski, Jeffrey

Rakos, Richard F

Read, J Don

Redding, Richard E

Redlich, Allison D

Reeve, Charlie L

Richards, Henry

Richardson, James

Robbennolt, Jennifer K

Roberts, Caton

Robicheaux, Tim*

Rose, Mary R

Rosenfeld, Barry

Ross, David

Rottman, David

Ruback, Richard B
Russano, Melissa B

Ryba, Nancy L

Saks, Michael

Salekin, Randy

Schneider, Kimberly

Schopp, Robert F

Schuller, Regina

Seelau, Sheila M

Semmler, Carolyn

Seto, Michael

Shaw, John

Shestowsky, Donna

Shuman, Daniel

Sinclair, H Colleen

Skeem, Jennifer L

Slobogin, Christopher

Smith, Amy

Sommers, Samuel

Spellman, Barbara A

Sporer, Siegfried L

Stalans, Loretta J

Steadman, Henry J

Steblay, Nancy K

Stevenson, Margaret*

Studebaker, Christina A

Terrance, Cheryl

Thompson, William C

Tredoux, Colin

Turtle, John

Tyler, Tom R

Vidmar, Neil

Vincent, Gina M

Vrij, Aldert

Walters, Glenn D

Weber, Nathan

Wells, Gary L

White, Larry

Zaragoza, Maria S

Zimmerman, Laura*

\footnotetext{
${ }^{*}$ Student Reviewer

Springer
} 